\title{
MR Compatible Surgical Assist Robot: System Integration and Preliminary Feasibility Study
}

\author{
Kiyoyuki Chinzei ${ }^{1}$, Nobuhiko Hata ${ }^{2}$, Ferenc A. Jolesz ${ }^{2}$, and Ron Kikinis ${ }^{2}$ \\ 1 Mechanical Engineering Laboratory, AIST, MITI \\ 1-2 Namiki, Tsukuba, 305-8564 Japan \\ chin@mel.go.jp (after Apr. 2001, chin@aist.go.jp) \\ http://www.mel.go.jp (after Apr. 2001, http://www.aist.go.jp) \\ 2 Department of Radiology, Brigham and Women's Hospital \\ Francis St. 75, Boston, MA 02115, USA \\ \{noby, jolesz, kikinis\}@bwh.harvard.edu \\ http://splweb.bwh.harvard.edu:8000
}

\begin{abstract}
A magnetic resonance (MR) compatible surgical assist robot system under preclinical evaluation is described. It is designed to coexist, and cooperate, with a surgeon, and to position and direct an axisymmetric tool, such as a laser pointer or a biopsy catheter. The main mechanical body is located above the head of the surgeon, and two rigid arms extend to the workspace. This configuration contributes to a small occupancy in the surgeon's workspace, and good MR compatibility.

The design of the robot is described. The MR compatibility is examined, and shows that there is no adverse effect on the imaging, even when the robot is in motion. Any heating effect was not evaluated, because a published study has revealed any effect is quite small. This robot system is carefully designed for safety and sterilization issues.
\end{abstract}

\section{Introduction}

The advantages of surgical robots and manipulators are well recognized in the clinical and technical community. Precision, accuracy, and the potential for telesurgery are the prime motivations in applying advanced robot technology in surgery $[1,2,3,4]$. Except for master-slave manipulators, which are controlled by human operators, surgical robots require trajectory planning, which, in practice, relies upon preoperative images. If the target organ is deformable, then the trajectory needs to be updated according to the magnitude of the deformation. Here, image-guided surgery is a natural solution.

Magnetic resonance imaging (MRI) has an excellent soft tissue discrimination, and a well-defined 3D coordinate reference system. An intraoperative MR scanner (Signa SP/i, GE Medical Systems, Milwaukee, WI, 0.5 Tesla) has been specifically designed to bring the power of MRI to the operating theater. It has a pair of parallel facing donut-shaped magnets, with an air gap of $560 \mathrm{~mm}$. (Fig. 1 left) Two surgeons can stand in the gap to access the patient. In the six years to February 2000, the authors' institute has recorded more than 500 cases using theintraoperative MR scanner $[5,6,7]$. 

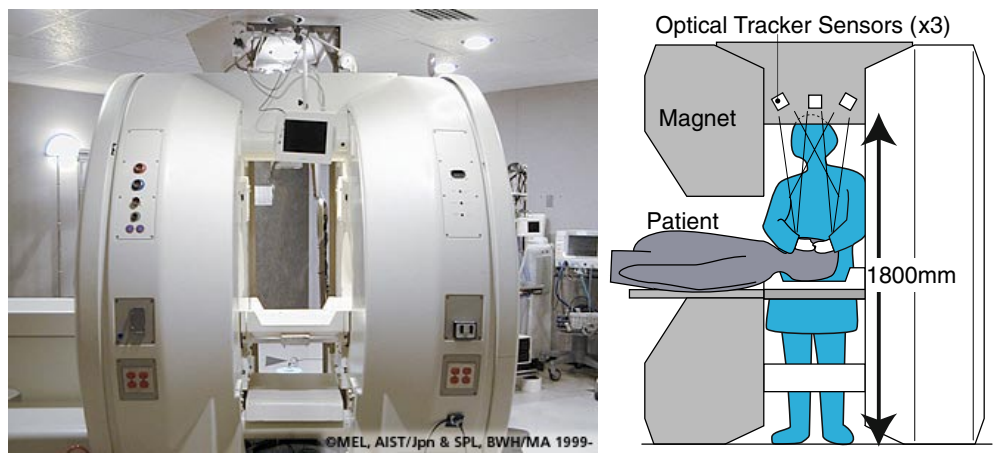

Fig. 1. The intraoperative MR scanner (left) and its profile with a patient and a medium height surgeon (right). Three visual sensors of the tracking device are installed above the imaging region. The sensor view has to be clear.

MR guidance of a surgical robot will be quite useful, as it can update the trajectory. However, an MR scanner is a highly restrictive environment for foreign objects, particularly for robots. Schenck has discussed the magnetic compatibility of MRI systems [8]. GE Medical Systems have issued a comprehensive and practical guideline regarding MR safety and compatibility [9], and Masamune has developed an MR compatible six degrees-of-freedom manipulator [10]. This was mainly built from plastics, and so suffered from a lack in rigidity. We have previously investigated the MR compatibility of mechanical parts, and have summarized MR compatible techniques, thereby showing the possibility of building a precise MR compatible mechanism [11].

In this paper, we demonstrate a unique configuration of a novel MR compatible robotic system for use in MR guided surgery. In Section 2.1, we introduce our target applications, and then define the design concept of the robot. Section 2 explains the unique configuration and the design of the robot system. In Section 3, the MR compatibility of the moving robot is validated. Safety and sterilization issues are discussed in Section 4.

\section{Materials and Methods}

The design of the robotic system will be introduced following the task definition. Two specific restrictions need to be overcome; (i) the layout of the robot needs to coexist, and cooperate with, the surgeon; and (ii) the design needs to be MR compatible.

\subsection{Task Definition}

The goal of our robot assist system is to enhance the surgeon's performance by accurate numerical control, and not to eject him or her from the surgical field. 
Therefore, the system must coexist, and cooperate, with the surgeon. Minimallyinvasiveness is an obvious requirement. The robot will hold a catheter or a laser pointer. In the former case, the robot will position and direct the catheter. Technically, the robot can perform the insertion; however, our current plan reserves this task for surgeon owing to ethical and legal considerations.

\subsection{Specific Restrictions in the MR Environment}

The intraoperative MR scanner requires two specific restrictions to the surgical robot, in addition to the standard requirements such as safety and sterilization policies.

Layout: The robot must coexist with the surgeon. However, when the patient is prepared, and the surgeons take their place, the available space for the robot is quite limited, particularly around the patient. In addition, the occlusion of the sensors of the optical tracker (Fig. 1, right) should be minimal. However, if the robot is placed some distance from the workspace, it will reduce the precision and the dynamic response.

MR compatibility: To enable the real-time tracking of the target position, the robot should be able to maneuver, even during imaging. The robot motion should not have any adverse effect on the imaging, and it should not be affected by the imaging process. This requires that the robot at least be made from paramagnetic materials. The further these are from the imaging region and the smaller volume they occupy, the better for MR compatibility. In addition, the robot should be MR safe. The MR safety of the robot requires that the machine should not unintentionally move from any magnetic attraction, and adverse electromagnetic side effects (e.g., leakage of, and heating by, eddy currents and RF pulses) should not occur.

To clear the workspace for the surgeon, the robot should be placed away from the surgical field. This also contributes to a better MR compatibility. However, there is a trade-off between space saving and mechanical performance.

\subsection{Configuration and Kinematics}

Figure 2 shows a schematic configuration of the robot. The actuators and the end effector are spatially separated. The main body, with all actuators, is located above the surgeon's head, and the end effector is attached at the ends of two long, rigid arms. The robot has five degrees-of-freedom, which define a polar coordinate system. Five degrees-of-freedom are sufficient to position and direct a catheter or a laser pointer, because these instruments are axisymmetric.

All axes in the main body are driven by linear motion mechanisms. The first three axes (X1, Y1, Z1) drive a rigid arm (Arm1) as well as the mechanism of remaining two axes (X2, Y2). These two axes drive the second rigid arm (Arm2). These arms are linked through two pivotal joints (P1, P2). P1 defines a point in the $3 \mathrm{D}$ coordinate system, and $\mathrm{P} 2$ travels in a $2 \mathrm{D}$ plane relative to $\mathrm{P} 1$. The 


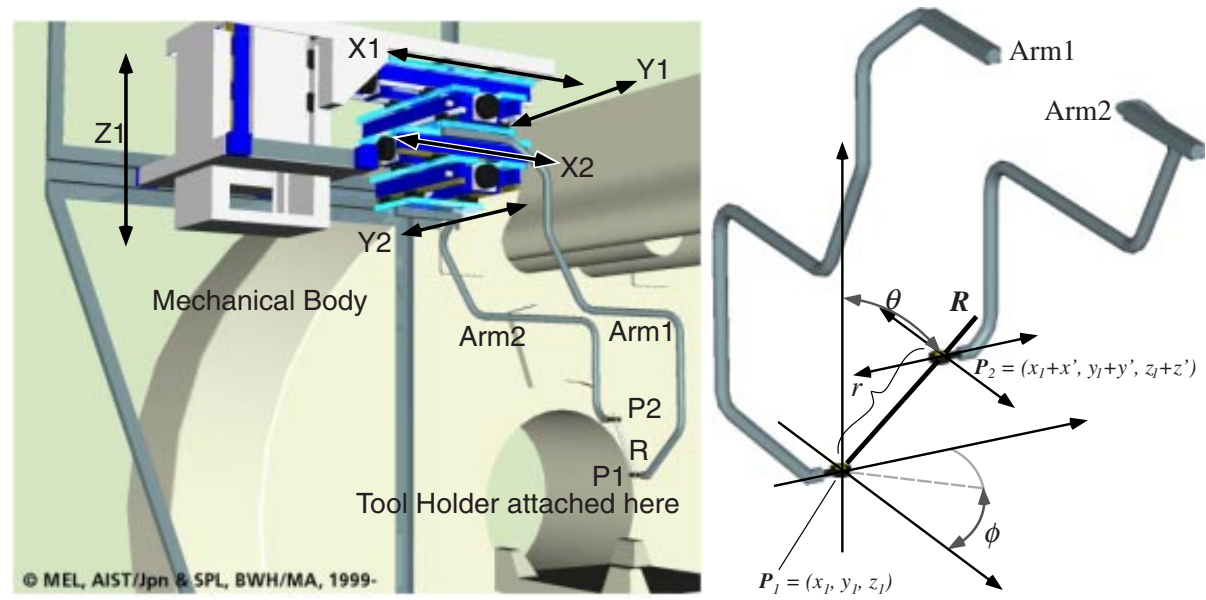

Fig. 2. The configuration of the robot. The mechanical body has 5 axes in total. All axes are driven by the linear motion mechanisms. Two rigid arms extend to the workspace. Arm 1 is actuated by X1, Y1 and Z1. Arm 2 is actuated by X2 and Y2. The ends of the arms are linked by two spherical joints P1, P2 and the sliding bar R. A tool holder is attached at P1. P1 moves in 3D space and P2 moves in a 2D plane relative to $\mathrm{P} 1$. The direction of the line segment between $\mathrm{P} 1$ and $\mathrm{P} 2, r$, is determined by the position of P2. Mathematically, it is a polar coordinate system whose origin is P1.

link between $\mathrm{P} 1$ and $\mathrm{P} 2, R$ in Fig. 2, is fixed to the inner ring of $\mathrm{P} 1$, whereas there is a sliding joint between $\mathrm{P} 2$ and $R$. This is to allow the distance between $\mathrm{P} 1$ and $\mathrm{P} 2, r$, to vary according to the position of P2.

The end effector, the tool holder, is attached to P1. For simplicity, here we will assume that the position of the tool holder is at $\mathrm{P} 1$, and its direction of travel is parallel to that of $R$. This is described in a polar coordinate system whose origin is at P1 (Eq. 1)

$$
\begin{aligned}
x^{\prime} & =r \cos \phi \sin \theta \\
y^{\prime} & =r \sin \phi \sin \theta \\
z^{\prime} & =r \cos \theta
\end{aligned}
$$

where $\mathrm{P} 1=(x 1, y 1, z 1), \mathrm{P} 2=\left(x 1+x^{\prime}, y 1+y^{\prime}, z 1+z^{\prime}\right),\left(z^{\prime}\right.$ is a constant $)$, and $r^{2}=x^{\prime 2}+y^{\prime 2}+z^{\prime 2}$. The direction $(\phi, \theta)$ is determined only by the relative position of $\mathrm{P} 2$ to $\mathrm{P} 1,\left(x^{\prime}, y^{\prime}, z^{\prime}\right)$. Therefore, the direction is independent of $\mathrm{P} 1$. When the tool holder is attached to P1 with an offset, its position is dependent on $(\phi, \theta)$.

\subsection{Design}

The hardware of the robot was assembled by Unitek Co. (Tsukuba, Japan) and partly by Drenak Co. (Machida, Japan.) The five degrees-of-freedom main body 
is composed of five linear motion tables. Each table unit has a ball screw and a pair of linear guides. These are made of either stainless steel (YHD50) or beryllium-copper; both materials have low magnetic susceptibility and a hard surface that can be used as a point-touch mechanism. The ball screws and linear guides made from YHD50 were manufactured by NSK Ltd. (Tokyo, Japan), and those made from beryllium-copper were manufactured by Koyo Seiko Co., Ltd. (Osaka, Japan.) The ball screw is supported by a pair of ball bearings made from silicon nitride $\left(\mathrm{Si}_{3} \mathrm{~N}_{4}\right)$ ceramics, manufactured by the above companies.

A non-magnetic (piezoelectric) ultrasonic motor, USR60-S3N, (Shinsei Kogyo Corp., Tokyo, Japan) directly drives the ball screw. Its maximum rotational torque is $0.5 \mathrm{Nm}$, and its holding torque is more than $0.7 \mathrm{Nm}$. A mechanical clutch is inserted between the motor and the ball screw to allow manual motion.

Each axis has the home and limit detector, and an incremental linear encoder. The linear encoder is customely designed, using glass grating patterns manufactured by Dynamic Research Corp. (Wilmington, MA). Its resolution is currently chosen to be $0.02 \mathrm{~mm}$. All signals are picked up by, and transferred via, fiber optic cables. The optoelectronic conversion circuits, as well as the other control and power circuits, are placed outside the scanner room for better noise immunity.

All parts of the robot were made from paramagnetic materials. The rigid arms, the frame structure of the vertical axis, and the attachment of the robot to the scanner were made from a titanium alloy. The frames of the horizontal axes were made from a polycarbonate resin. All the screws were made from a titanium alloy or brass, to avoid any attraction of loose screws from the magnet.

The pivotal joints, P1 and P2, were KBRM-10 Rod End Bearings (IGUS $\mathrm{GmbH}$, Germany), and were all made from plastics, and the connecting link, $R$, was made from acrylic resin. As these are insulating materials, the leakage of the eddy current and RF pulses can be safely avoided. Each arm can be divided into three pieces. The end pieces can fit in a typical autoclave tray, whose internal dimensions are approximately $450 \times 80 \times 200 \mathrm{~mm}$.

Cooperation between the robot control, MRI, and 3D position tracking is implemented by the object distributed, server-client model [12]. Currently there are three modules: (i) a robot hardware module; (ii) a Modular Robot Control (MRC) developed at Johns Hopkins University; and (iii) Slicer3D modules (Image processing/surgical planning) [13]. The details of these are to be found in the given references.

\subsection{Evaluation of MR Compatibility}

We have examined the loss of homogeneity of the magnetic field, and the signalto-noise ratio (SNR) of the image. There are several possible interactions when the robot is located in, and is maneuvering in, the MR environment. The presence of, and motion of, the robot can distort, or shift, the image by decreasing the homogeneity of the magnetic field, and these can also affect the image SNR. The leakage of the induced current can be safely neglected by the insulating devices 
as described above. The heaing effect has been proved to be minor as shown by the previous work [14].

A spherical phantom (diameter $=280 \mathrm{~mm}$ ) was imaged using the imager. This contained $\mathrm{CuSO}_{4}$ solution, which gave a delta-function shape resonance spectrum in an ideal, homogenous magnetic field. The inhomogeneity was defined by the diversity of the observed spectrum. The SNR was calculated using the following equation (Eq. 2).

$$
S N R=\text { Pcenter } / \text { SDcorner . }
$$

where Pcenter is the mean value of the $40 \times 40$ pixel area at the center of the image, and SDcorner is the standard deviation of the $40 \times 40$ pixel area in the lower right corner of the image. The sequence was the Spin Echo, TE/TR $=85 / 220 \mathrm{~ms}$, and the receiver bandwidth was $62.5 \mathrm{kHz}$. These protocols are described in [9]. The robot repeated a simple Y2 axis motion, which was the most adjacent axis to the imaging region. The control data were obtained by the same phantom without the robot.

\section{Results}

The construction of a Mark 1 version of the robot system has already been completed. The system is currently in the software tuning, and the preclinical evaluation stages.
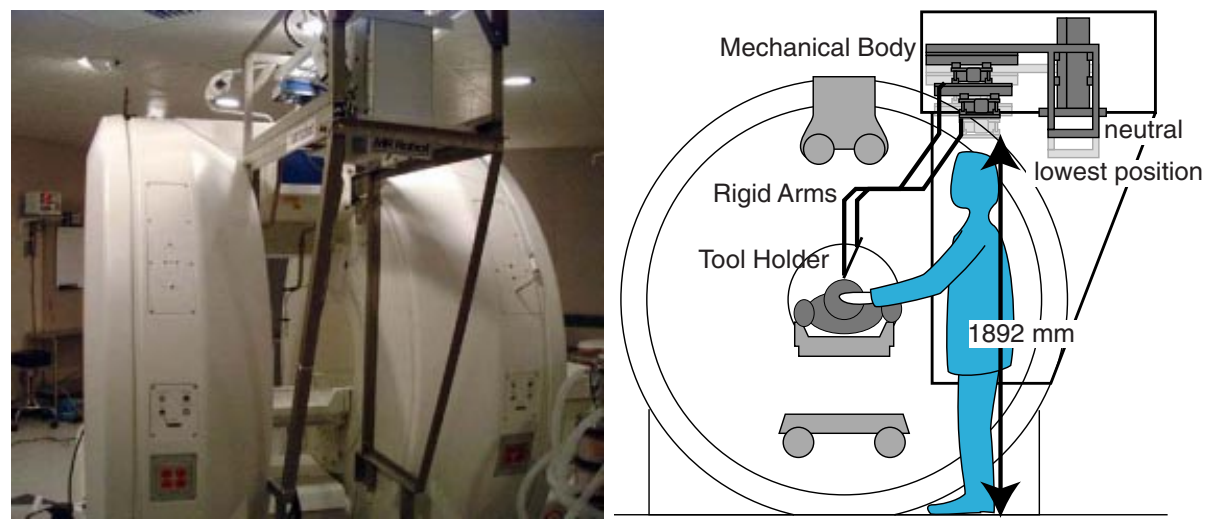

Fig. 3. Constructed robot attached with the intraoperative MR scanner (left) and the profile of the workspace (right). The moving part clears the workspace for the surgeon.

\subsection{Configuration}

Figure 3 shows the constructed robot installed with the intraoperative MRI and the profile of the workspace. 
When the vertical axis is at the lowest position, the moving part's lowest height (except the arms and part of the vertical axis, Z1) is $1892 \mathrm{~mm}$ from the floor (the bottom of the Y2 axis, Fig. 3, right). The arms are bent so that they do not collide with the scanner.

\subsection{MR Compatibility}

The magnetic field inhomogeneity values are listed in Table 1 . The inhomogeneity value was observed to be 0.53 when the robot was in motion. This is better than that of a clinically-used stereotactic frame, or of the human body itself. Therefore, the effect on the homogeneity of the magnetic field was negligible.

Table 1. Obtained inhomogeneity values. The smaller value is the greater homogeneity.

\begin{tabular}{l|c} 
Inhomogeneity & $(\mathrm{ppm})$ \\
\hline Spherical phantom, without robot (baseline) & 0.45 \\
\hline Spherical phantom, with moving robot & 0.53 \\
\hline $\begin{array}{l}\text { Spherical phantom, with an 'MR compatible' Mayfield } \\
\text { stereotactic frame }\end{array}$ & 0.9 \\
\hline Human volunteer & ca. 1.4
\end{tabular}

The observed signal-to-noise ratio (SNR) loss was $1.6 \%$ to $1.8 \%$. As an SNR loss up to $10 \%$ is acceptable, the observed SNR was in the negligible range. Figure 4 shows an image of the spherical phantom with, and without, the robot.
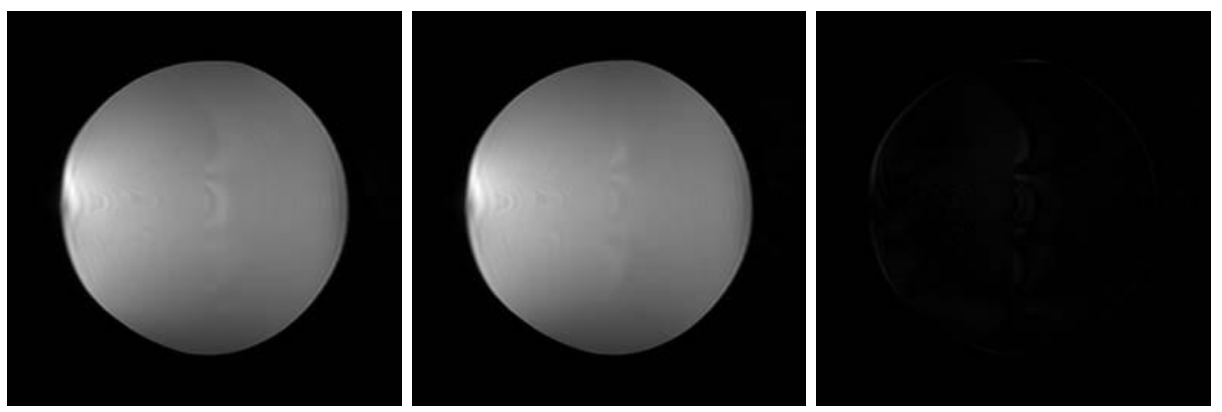

Fig. 4. Images of the spherical phantom when the robot was not installed (left) and when one axis of the robot was in motion (center). The subtraction of these images showed no shift (right).

\section{Discussion}

This configuration has several advantages:

- The mechanical body is positioned 1-2 m away from the center of the imaging volume. It also relaxes the MR compatibility criteria. 
- The kinematics and inverse kinematics are simple. It forms a polar coordinate system, and the position and the direction can be independently determined by the position of the pivoltal joints P1 and P2. When the surgeon wants to move the end effector numerically, he or she can easily predict the motion of the robot.

- The arms and end effector are detachable, exchangeable, and sterilizable. This design also allows for the selection of a suitable arm shape, according to the desired procedure.

- The arms have no rotational joint and no actuator to sustain. Without these, the robot has the slender arms that save the precious workspace. The parallel-link mechanism has the similar advantage, however, it usually demands a large motion space. However, Koseki and the author are proposing a combination of a leverage and parallelepiped mechanisms (LPM) [15]. LPM mirrors the motion of the robot at a distance, therefore it has a potential to be a break-through to relax the specific restrictions in the MR environment.

On the other hand, the end effector needs to be light and axisymmetric, such as a catheter needle. As the needle insertion is one of common tasks in many minimally invasive procedures, this design does not lose a generality.

\subsection{Safety and Sterilization Issues}

In general, it is the surgeon's responsibility to avoid a collision, as there is no reliable, and universal, automated method to address this issue. Therefore, the simplicity of the kinematics, in particlar, the independency of the parameters, is an important factor in helping the surgeon to understand the motion of the robot.

Brakes are not necessary, as the motor's holding torque is strong, and the robot maintains its posture even in the event of a power cut. The noise immunity of the sensors is good, since the sensors use fiber optics to carry the signals.

The rigid arms can be divided into shorter pieces. The end piece is autoclavable. The other part of the arms, and the mechanical body, can be draped. The pivot joints and other plastics parts can be applied EOG sterilization.

\subsection{An Example of Application}

One of the good examples of this robot system is the needle navigation in the brachytherapy of prostate cancer. This is a minimally invasive outpatient radiotherapy that delivers an internalized radioactive source to the cancer. A number of small iodine-125 $\left({ }^{125} \mathrm{I}\right)$ radiation seeds are placed using catheters, under the guidance of MRI. The authors' institute has pioneered the use of MR guided brachytherapy of prostate cancer since 1997 [16].

This procedure implants 50 to 120 seeds by 12 to 20 catheter insertions, according to a preoperative seeding plan. It is a skilful, and hard, task for human operator, whereas it will be a good application for the robot. 


\subsection{Impact of the MR Guided Robotic Assist}

Modern medicine has rich sources of information regarding the state of health of a patient. High quality three dimensional images (e.g., MRI) transfer the real world (patient) into the virtual world. We have developed computer models for virtual manipulation for surgical planning. In contrast, the transfer from the virtual to the real world (i.e., the operating theater) has been mostly limited to visual assist. Here, the flow of online information is unidirectional and incomplete. Surgical robots will be the means of physical assistance, and create a bi-directional flow of online information. The impact of the combination of the intraoperative MRI, and the MR compatible robot, will be even greater, because it will bring the possibility of near real-time processing of the virtual and real worlds in a bi-directional manner.

\section{Conclusion}

An MR compatible surgical assist robot is in the preclinical evaluation stage. It is designed to position and direct an axisymmetric tool, such as a laser pointer or a biopsy catheter. An example of its application is in MR guided brachytherapy of prostate cancer. It has a generality, because catheter insertion is a common procedure in many other operations.

To be MR compatible, and to fit into the reduced space of the intraoperative MRI, the robot has a unique configuration. The robot has five degreesof-freedom. All the mechanical axes are in linear motions. The end effector is attached at the end of two long rigid arms. The end effector forms a polar coordinate system. The robot is made from a titanium alloy and plastic and is driven by non-magnetic ultrasonic motors. Fiber optics carry the signals from the sensor heads to circuit boards that are isolated outside the MRI room for noise immunity.

The robot shows excellent MR compatibility. Its motion does not appear to have any adverse effect on the imaging, and the robot is not affected by the imaging process. Other possible events (e.g., heating) are considered to be negligible from the isolation of the design, and from previous studies in literature.

\section{Acknowledgment}

In Japan, the project has been funded by MITI, "Study of Open MRI-Guided Diagnosis/Treatment Concurrent System", and "Research on MR compatible active holder for surgical assistance".

In the USA, the project has been funded by NSF Engineering Research Laboratory "Computer Integrated Surgical Systems and Technology" \#9731748, and "MR Guided Therapy" 3P01CA67165-03S1 funded by NIH.

The authors would gracefully acknowledge the assists of Mr. Dan Kacher, Mr. Oliver Schorr and Dr. Toshikatsu Washio to conduct the experiments. 


\section{References}

[1] Villotte, N., Glauser, D., Flury, P., et.al.: Conception of Stereotactic Instruments for the Neurosurgical Robot Minerva. in proc IEEE ICRA (1992) 1089-90.

[2] Taylor, R.H., Mitterlstadt, B.D., Paul, H.A., et.al.: An Image-Directed Robotic System for Precise Orthopedic Surgery. in Taylor, R.H., et.al. (eds) ComputerIntegrated Surgery : Technology and Clinical Applications, MIT Press (1995) 37995.

[3] Sackier, J.M., Wang, Y.: Robotically Assisted Laparoscopic Surgery: from Concept to Development in Taylor, R.H., et.al. (eds) Computer-Integrated Surgery : Technology and Clinical Applications, MIT Press (1995) 577-80.

[4] Schenker, P.S., Das, H., Ohm, T.R.: A New Robot for High Dexterity Microsurgery. in proc CVRMed95, Lecture Notes Computer Science, 905, SpringerVerlag (1995) 115-22.

[5] Schenck, J.F., Jolesz, F.A., Roemer, P.B., et. al.: Superconducting OpenConfiguration MR Imaging System for Image-Guided Therapy. Radiology, 195(3) (1995) 805-14.

[6] Silverman, S.G., Collick, B.D., Figueira, M.R., et.al.: Interactive MR-guided biopsy in an open-configuration MR imaging system. Radiology, 197 (1995) 175-81.

[7] Hata, N., Morrison, P.R., Kettenbach, J., Black, P., Kikinis, R., Jolesz, F.A.: Computer-assisted Intra-Operative Magnetic Resonance Imaging Monitoring of Interstitial Laser Therapy in the Brain: A Case Report. J Biomedical Optics, 3(3) (1998) 304-11.

[8] Schenck, J.F.: The role of magnetic susceptibility in magnetic resonance imaging: MRI magnetic compatibility of the first and second kinds. Med Phys, 23(6) (1996) $815-50$.

[9] GE Medical Systems (ed): MR Safety and MR Compatibility: Test Guidelines for Signa SP ${ }^{\mathrm{TM}}$. www.ge.com/medical/mr/iomri/safety.htm, 1997.

[10] Masamune, K., Kobayashi, E., Masutani, Y., et.al.: Development of an MRI compatible Needle Insertion Manipulator for Stereotactic Neurosurgery. J Image Guided Surgery, 1 (1995) 242-8.

[11] Chinzei, K., Kikinis, R., Jolesz, F.A.: MR Compatibility of Mechatronic Devices: Design Criteria. in proc MICCAI'99, Lecture Notes in Computer Science, 1679 (1999) 1020-31.

[12] Schorr, O., Hata N., Bzostek, A., Kumar, R., Burghart, C., Taylor, R.H., Kikinis, R.: Distributed Modular Computer-Integrated Surgical Robotic Systems: Architecture for Intelligent Object Distribution. in proc MICCAI 2000 (printing), Pittsburgh PA (2000).

[13] Gering D., Nabavi, A., Kikinis, R., Eric, W., Grimson, L., Hata, N., et.al.: An Integrated Visualization System for Surgical Planning and Guidance using Image Fusion and Interventional Imaging. in proc. MICCAI '99, Lecture Notes in Computer Science, 1679, Springer-Verlag, Berlin, Heiderberg, New York (1999) 809-19.

[14] Buchili, R., Boesiger, P., Meier, D.: Heating Effects of Metallic Implants by MRI Examinations. Magnet Reson Med, 7 (1988) 255-61.

[15] Koseki, Y., Chinzei, K., Koyachi, N., Arai, T.: Robotic Assist for MR-Guided Surgery Using Leverage and Palallelepiped Mechanism. in proc MICCAI 2000 (printing), Pittsburgh PA (2000).

[16] D'Amico, A.V., et al.: Real-time magnetic resonance image-guided interstitial brachytherapy in the treatment of select patients with clinically localized prostate cancer. Int J Radiat Oncol Biol Phys, 42(3) (1998) 507-15. 